Supplement of

\title{
Analysis of atmospheric ammonia over South and East Asia based on the MOZART-4 model and its comparison with satellite and surface observations
}

Pooja V. Pawar et al.

Correspondence to: Sachin D. Ghude (sachinghude@ tropmet.res.in)

The copyright of individual parts of the supplement might differ from the article licence. 
Table S1. Location of $\mathrm{NH}_{3}$ measurement sites from the air quality monitoring network operated by the Central Pollution Control Board, India (see also Figure 3)

\begin{tabular}{|c|c|c|c|c|c|}
\hline Site No. & Location name & Latitude & Longitude & Elevation (m) & Area \\
\hline 1 & Adarsh Nagar & 26.8754 & 75.8167 & 433 & Rural \\
\hline 2 & Devas & 22.9688 & 76.0636 & 563 & Rural \\
\hline 3 & Ajmer & 26.4727 & 74.6415 & 494 & Urban \\
\hline 4 & Nashik & 20.0073 & 73.7762 & 580 & Urban \\
\hline 5 & Visakhapatnam & 17.72 & 83.3 & 26 & Urban \\
\hline 6 & Ujjain & 23.1793 & 75.7849 & 497 & Urban \\
\hline 7 & Patiala & 30.3448 & 76.3708 & 256 & Urban \\
\hline 8 & Aurangabad & 19.8406 & 75.2466 & 521 & Urban \\
\hline 9 & Alwar & 27.5591 & 76.6021 & 276 & Urban \\
\hline 10 & Howrah & 22.5707 & 88.3008 & 8 & Coastal \\
\hline 11 & Kerela & 8.5141 & 76.9477 & 23 & Urban \\
\hline 12 & Vijaywada & 16.5064 & 80.632 & 25 & Urban \\
\hline 13 & Udaipur & 24.589 & 73.7022 & 575 & Urban \\
\hline 14 & Ratnapura & 31.029 & 76.5734 & 274 & Rural \\
\hline 15 & Bhiwadi & 28.207 & 76.8577 & 268 & Industrial \\
\hline 16 & Amravati & 16.5151 & 80.5182 & 14 & Rural \\
\hline 17 & Pithampur & 22.6248 & 75.6752 & 616 & Rural \\
\hline 18 & Mandideep & 23.099 & 77.505 & 445 & Industrial \\
\hline 19 & Kota & 25.136 & 75.8247 & 297 & Urban \\
\hline 20 & Lucknow & 26.834 & 80.8917 & 126 & Urban \\
\hline 21 & Kolkata & 22.5449 & 88.3425 & 18 & Urban \\
\hline 22 & Singrauli & 24.0886 & 82.6478 & 287 & Rural \\
\hline 23 & Delhi1 & 28.8229 & 77.102 & 212 & Urban \\
\hline 24 & Delhi2 & 28.6508 & 77.3152 & 224 & Urban \\
\hline
\end{tabular}




\begin{tabular}{|c|c|c|c|c|c|}
\hline 25 & Delhi3 & 28.6687 & 77.23 & 218 & Urban \\
\hline 26 & Delhi4 & 28.7762 & 77.0511 & 218 & Industria \\
\hline 27 & Delhi5 & 28.4997 & 77.2671 & 257 & Rural \\
\hline 28 & Delhi6 & 28.7501 & 77.1177 & 214 & Urban \\
\hline 29 & Delhi7 & 28.5713 & 77.0644 & 220 & Urban \\
\hline 30 & Delhi8 & 28.6803 & 77.2012 & 226 & Urban \\
\hline 31 & Delhi9 & 28.6317 & 77.2494 & 230 & Urban \\
\hline 32 & Delhi10 & 28.7255 & 77.1587 & 225 & Urban \\
\hline 33 & Delhi11 & 28.6687 & 77.1599 & 217 & Urban \\
\hline 34 & Delhi12 & 28.6124 & 77.2351 & 204 & Urban \\
\hline 35 & Delhi13 & 28.6338 & 77.198 & 219 & Urban \\
\hline 36 & Delhi14 & 28.6823 & 77.0349 & 217 & Urban \\
\hline 37 & Delhi15 & 28.6072 & 76.9459 & 218 & Urban \\
\hline 38 & Delhi16 & 28.6072 & 76.8408 & 212 & Urban \\
\hline 39 & Delhi17 & 28.6644 & 77.1704 & 238 & Urban \\
\hline 40 & Delhi18 & 28.5393 & 77.2687 & 233 & Urban \\
\hline 41 & Delhi19 & 28.6287 & 77.2946 & 220 & Urban \\
\hline 42 & Delhi20 & 28.6637 & 77.1196 & 22 & Urban \\
\hline 43 & Delhi21 & 28.632 & 77.1555 & 219 & Urban \\
\hline 44 & Delhi22 & 28.5646 & 77.167 & 225 & Urban \\
\hline 45 & Delhi23 & 28.7406 & 77.0577 & 212 & Urban \\
\hline 46 & Delhi24 & 28.5438 & 77.331 & 201 & Urban \\
\hline 47 & Delhi25 & 28.5504 & 77.2116 & 222 & Urban \\
\hline 48 & Delhi26 & 28.7373 & 77.2274 & 203 & Urban \\
\hline 49 & Delhi27 & 28.5062 & 77.2492 & 243 & Urban \\
\hline 50 & Delhi28 & 28.6688 & 77.3131 & 207 & Urban \\
\hline 51 & Delhi29 & 28.6981 & 77.1517 & 215 & Urban \\
\hline
\end{tabular}




\begin{tabular}{|c|c|c|c|c|c|}
\hline 52 & Delhi30 & 28.9575 & 77.2723 & 211 & Urban \\
\hline 53 & Delhi31 & 28.407 & 77.8498 & 197 & Urban \\
\hline 54 & Delhi32 & 28.694 & 77.455 & 226 & Urban \\
\hline 55 & Delhi33 & 28.6679 & 77.4498 & 208 & Urban \\
\hline 56 & Delhi34 & 28.8253 & 78.7213 & 193 & Urban \\
\hline 57 & Delhi35 & 29.4677 & 77.7116 & 245 & Urban \\
\hline 58 & Hyderabad1 & 17.4567 & 78.3264 & 580 & Rural \\
\hline 59 & Hyderabad2 & 17.5111 & 78.2752 & 544 & Rural \\
\hline 60 & Hyderabad3 & 17.5325 & 78.1849 & 545 & Industrial \\
\hline 61 & Hyderabad4 & 17.3507 & 78.4513 & 505 & Urban \\
\hline 62 & Bengaluru1 & 12.9568 & 77.5397 & 851 & Urban \\
\hline 63 & Bengaluru2 & 12.9135 & 77.5951 & 917 & Urban \\
\hline 64 & Bengaluru3 & 12.9756 & 77.6035 & 923 & Urban \\
\hline 65 & Bengaluru4 & 12.9172 & 77.5834 & 921 & Urban \\
\hline 66 & Bengaluru5 & 13.029 & 77.5197 & 909 & Urban \\
\hline 67 & Bengaluru6 & 12.9177 & 77.6238 & 882 & Urban \\
\hline 68 & Jaipur1 & 26.916 & 75.8017 & 435 & Urban \\
\hline 69 & Jaipur2 & 26.9503 & 75.801 & 470 & Urban \\
\hline
\end{tabular}


Table S2. Location of $\mathrm{NH}_{3}$ measurement sites from the Nationwide Nitrogen Deposition Monitoring Network (NNDMN) operated by China Agricultural University, China (see also Figure 3)

\begin{tabular}{|c|c|c|c|c|c|}
\hline $\begin{array}{l}\text { Site } \\
\text { No. }\end{array}$ & Location name & Latitude & Longitude & $\begin{array}{l}\text { Elevation } \\
\text { (m) }\end{array}$ & Area \\
\hline 1 & China Agricultural University & 40.02 & 116.28 & 50 & Urban \\
\hline 2 & Zhengzhou & 34.75 & 113.37 & 167 & Urban \\
\hline 3 & Dalian & 38.92 & 121.58 & 18 & Urban \\
\hline 4 & Nanjing & 31.84 & 118.85 & 9 & Urban \\
\hline 5 & Baiyun & 23.16 & 113.27 & 16 & Urban \\
\hline 6 & Wenjiang & 30.55 & 103.84 & 477 & Urban \\
\hline 7 & Shangzhuang & 40.11 & 116.20 & 44 & Rural \\
\hline 8 & Baoding & 38.85 & 115.48 & 15 & Rural \\
\hline 9 & Quzhou & 36.78 & 114.94 & 44 & Rural \\
\hline 10 & Yangqu & 38.05 & 112.89 & 1276 & Rural \\
\hline 11 & Zhumadian & 33.02 & 114.05 & 69 & Rural \\
\hline 12 & Yangling & 34.31 & 108.01 & 554 & Rural \\
\hline 13 & Yucheng & 36.94 & 116.63 & 24 & Rural \\
\hline 14 & Gongzhuling & 43.53 & 124.83 & 201 & Rural \\
\hline 15 & Lishu & 43.36 & 124.17 & 129 & Rural \\
\hline 16 & Wuwei & 38.07 & 102.60 & 1493 & Rural \\
\hline 17 & Wuxue & 30.01 & 115.79 & 16 & Rural \\
\hline 18 & Taojiang & 28.61 & 111.97 & 130 & Rural \\
\hline 19 & Fengyang & 32.88 & 117.56 & 66 & Rural \\
\hline 20 & Zhanjiang & 21.26 & 110.33 & 24 & Rural \\
\hline 21 & Fuzhou & 26.17 & 119.36 & 432 & Rural \\
\hline
\end{tabular}




\begin{tabular}{llllll}
22 & Fenghua & 29.61 & 121.53 & 34 & Rural \\
23 & Ziyang & 30.13 & 104.63 & 360 & Rural \\
24 & Yanting & 31.28 & 105.47 & 506 & Rural \\
25 & Jiangjin & 29.06 & 106.18 & 292 & Rural \\
26 & Lingshadao & 35.77 & 120.18 & 0 & Coastal \\
27 & Changdao & 37.93 & 120.75 & 59 & Coastal \\
28 & Duolun & 42.20 & 116.49 & 1239 & Grassland \\
29 & Bayingbuluke & 42.88 & 83.71 & 2468 & Grassland \\
30 & Feiyue & 28.56 & 113.34 & 77 & Forest \\
31 & Huinong & 28.52 & 113.41 & 96 & Forest \\
32 & Xishan & 28.61 & 113.31 & 230 & Forest \\
\hline
\end{tabular}




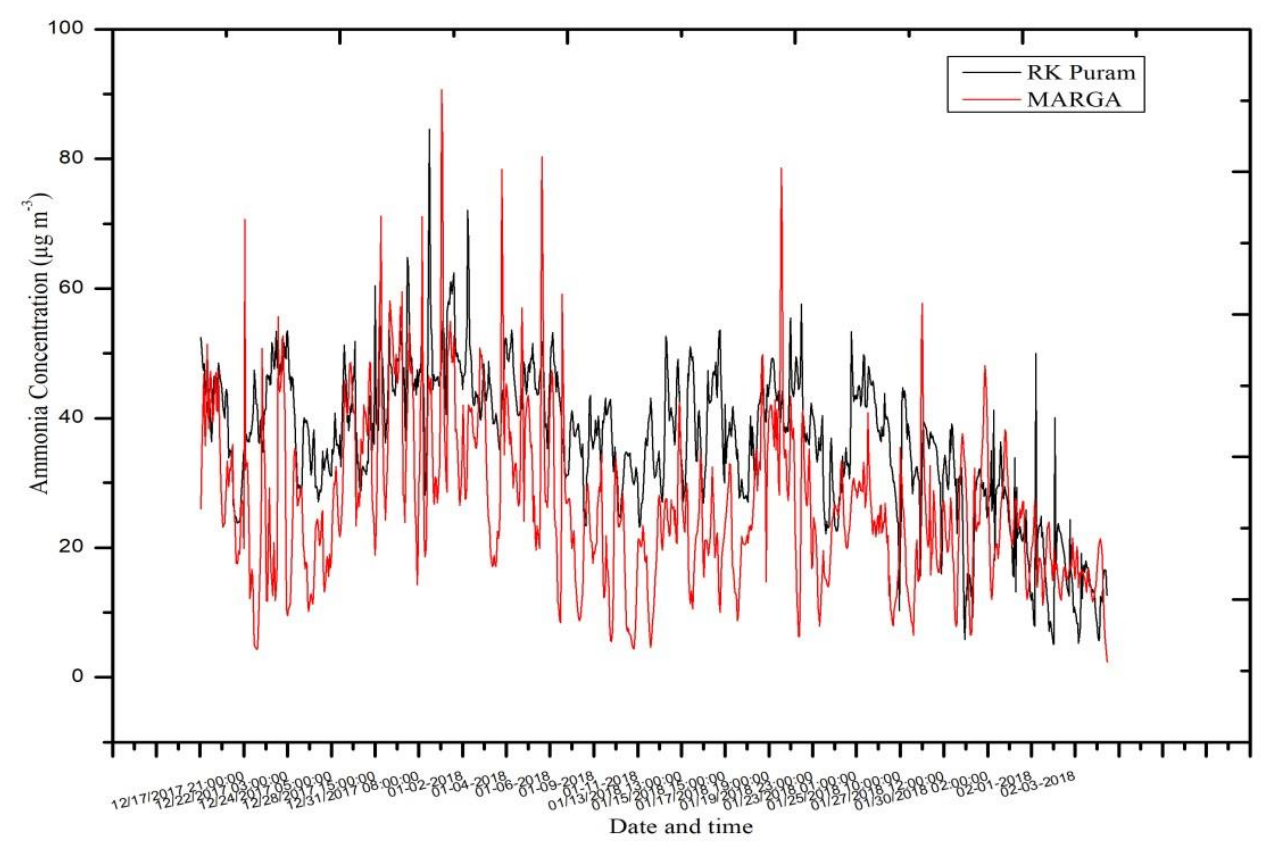

Figure S1. Comparison of $\mathrm{NH}_{3}\left(\mu \mathrm{g} \mathrm{m}^{-3}\right)$ concentration from MARGA instrument with RK Puram (CPCB) station 

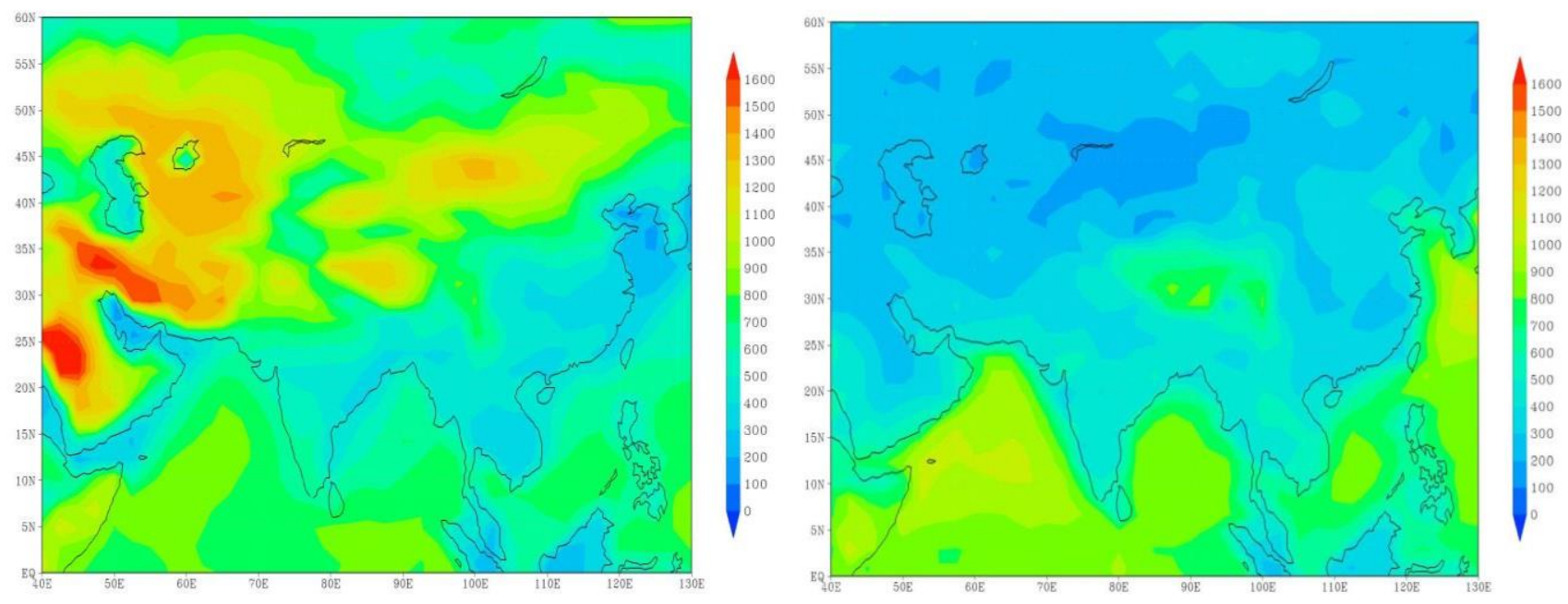

Figure S2. MOZART-4 model estimate of Planetary boundary layer height (PBLH) (m) during summer (JJA) season (left) and during winter (DJF) season (right). 


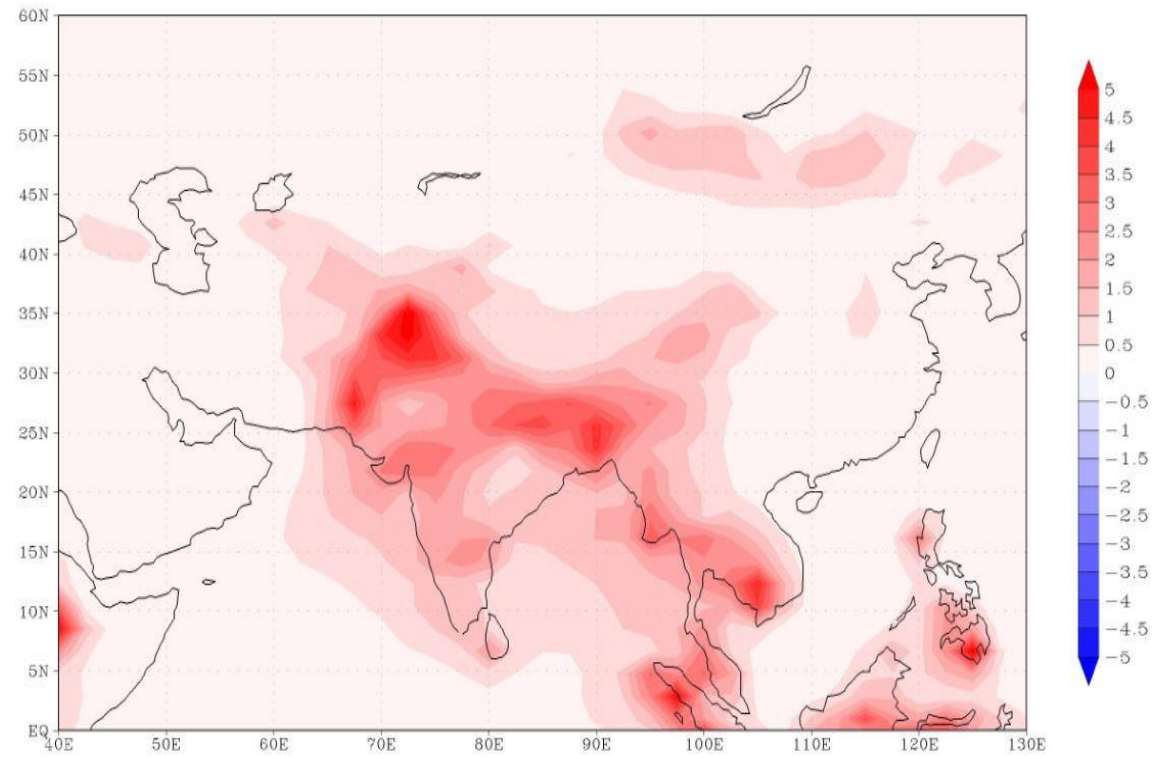

Figure S3. MOZART-4 model estimate of annual averaged $\mathrm{NH}_{3} / \mathrm{NH}_{4}$ ratio $\left(\times 10^{9} \mathrm{ppb}\right)$ over Asia. 

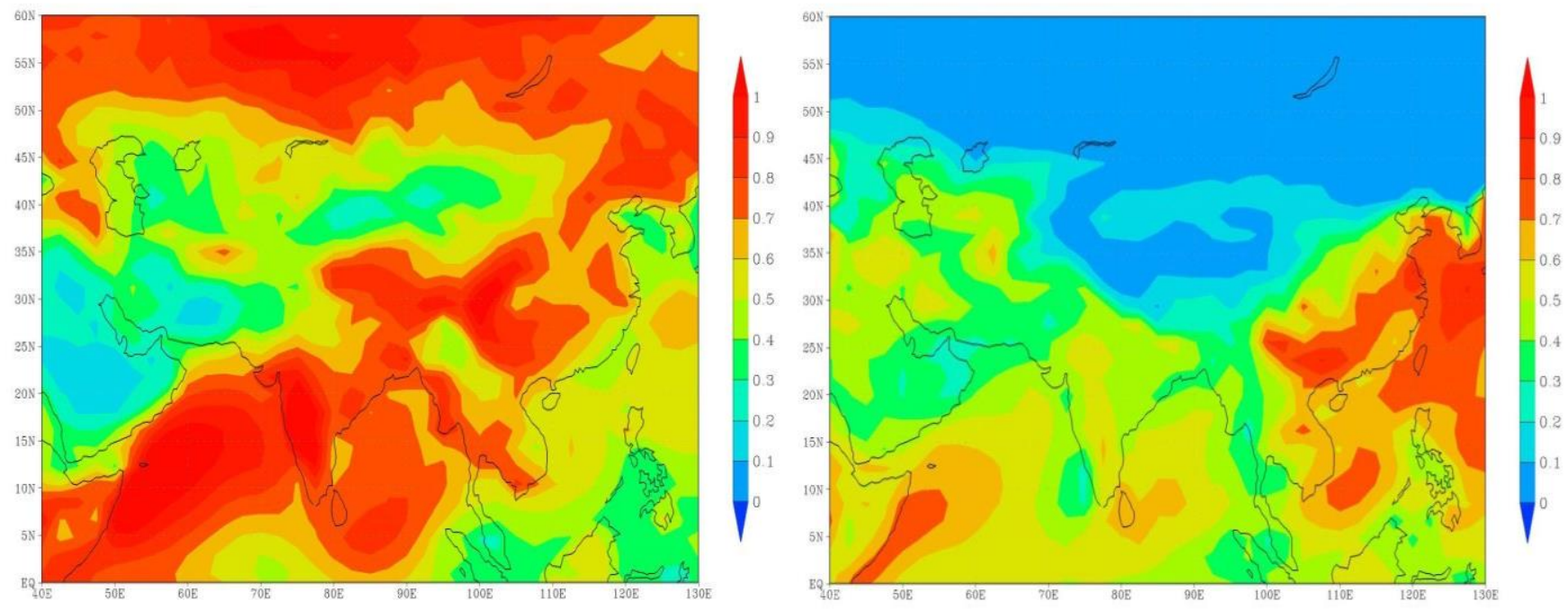

Figure S4. MOZART-4 model estimate of dry deposition velocity ( $\mathrm{cm} \mathrm{s}^{-1}$ ) during summer (JJA) season (left) and during winter (DJF) season (right). 

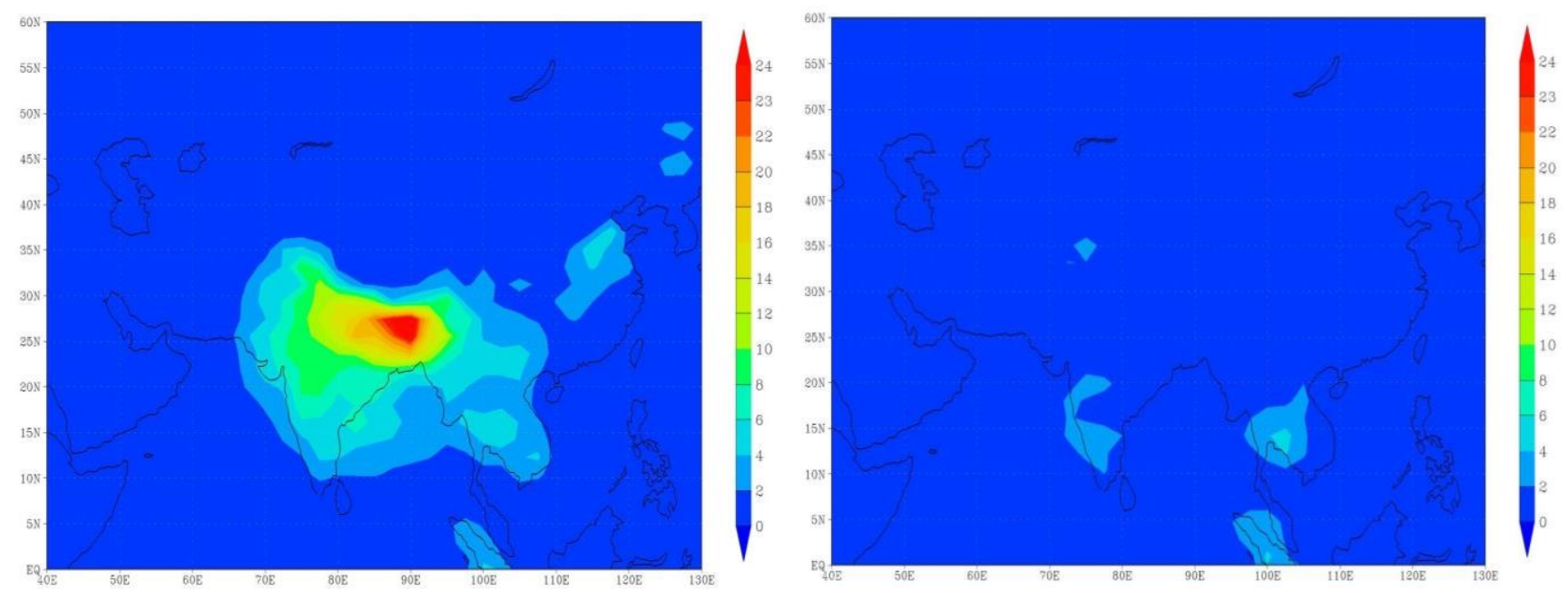

Figure S5. MOZART-4 model estimate of $\mathrm{NH}_{3}$ wet deposition flux $\left(\times 10^{-9} \mathrm{~kg} \mathrm{~m}^{-2} \mathrm{~s}^{-1}\right)$ during summer (JJA) season (left) and during winter (DJF) season (right). 

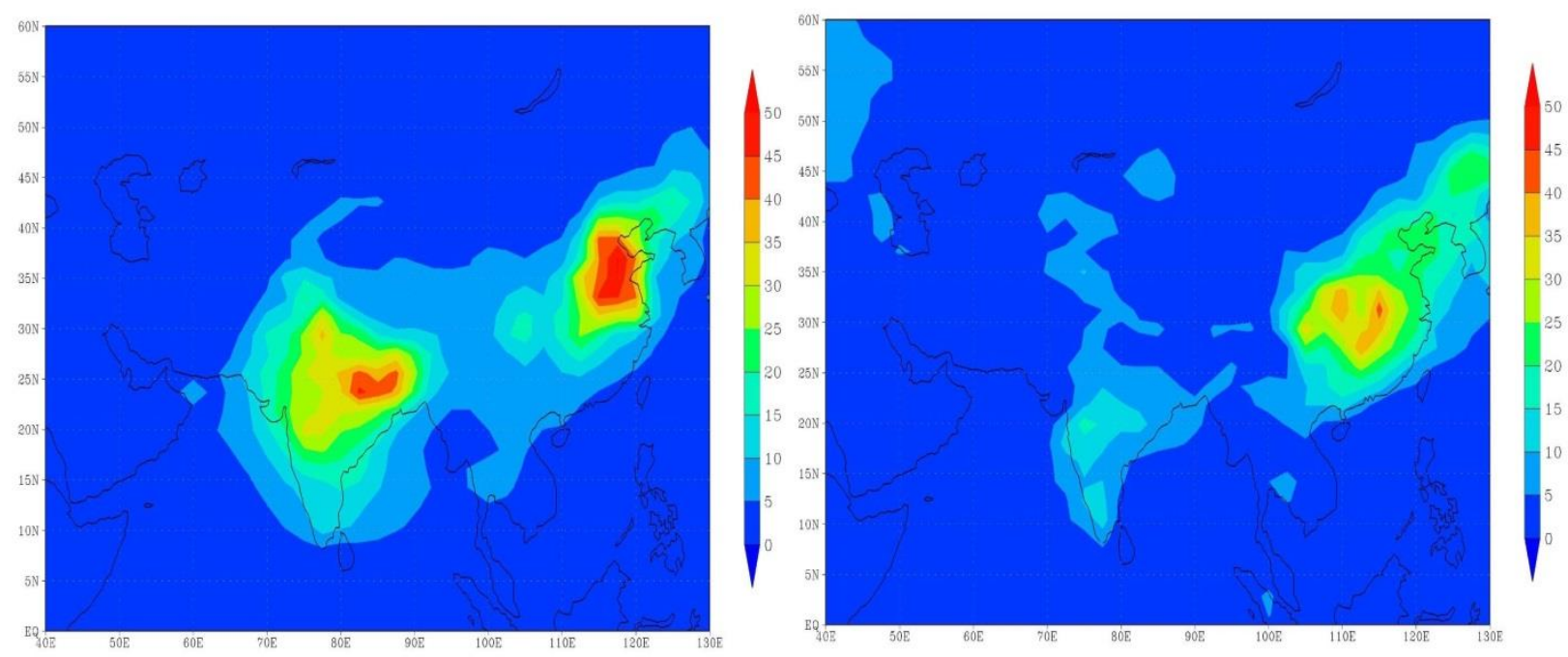

Figure S6. MOZART-4 model estimate of $\mathrm{NH}_{3} \mathrm{NO}_{3}$ wet deposition flux $\left(\times 10^{-9} \mathrm{~kg} \mathrm{~m}^{-2} \mathrm{~s}^{-1}\right)$ during summer (JJA) season (left) and during winter (DJF) season (right) 


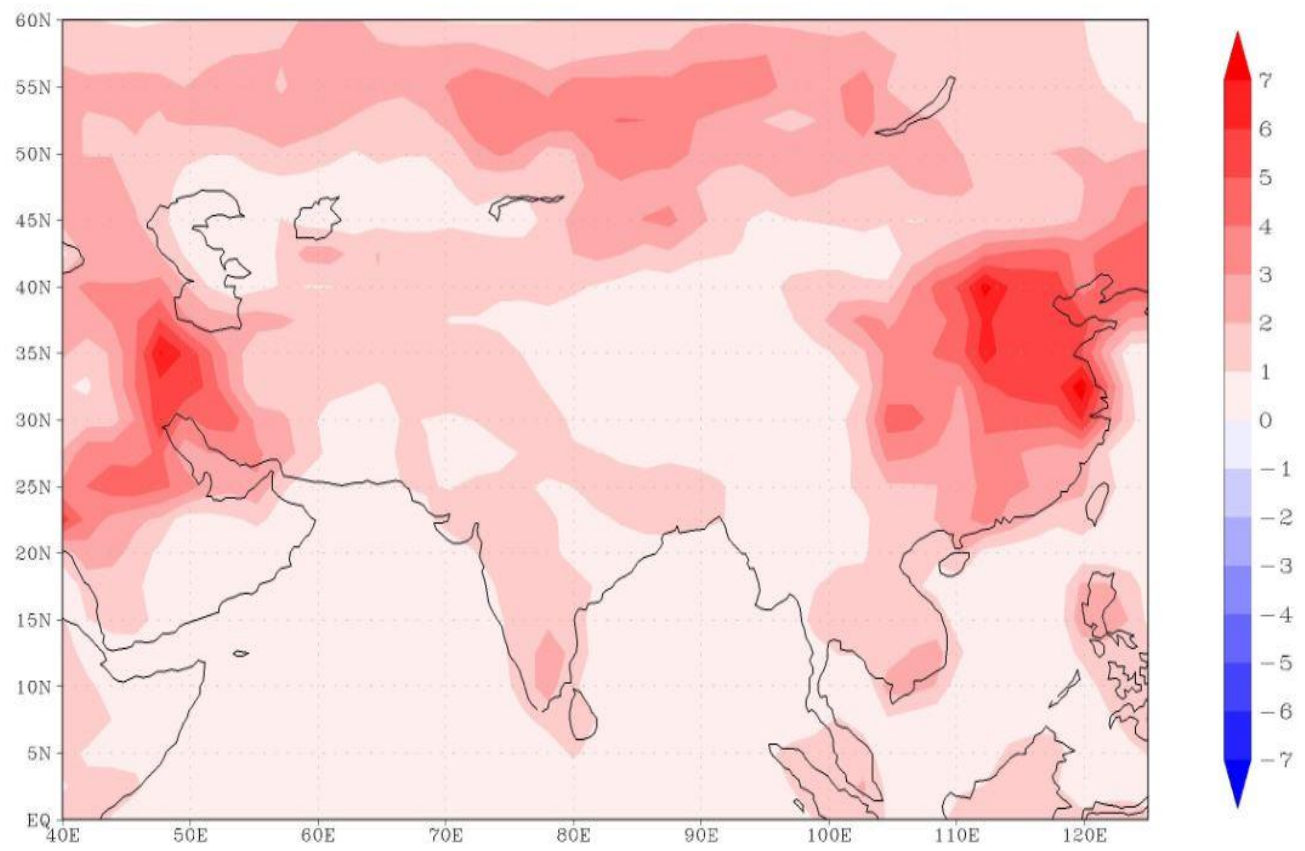

Figure S7. MOZART-4 model estimate of annual averaged $\mathrm{NH}_{3}$ total emissions $/ \mathrm{NH}_{3}$ total column ratio $\left(\times 10^{-5} \mathrm{~S}^{-1}\right)$ over $^{-}$ Asia. 